\title{
Awakening sleeper cells: a narrative review on bacterial magic spot synthetases as potential drug targets to overcome persistence
}

\author{
Vimal Venu Veetilvalappil $^{1}$ (D) Jesil Mathew Aranjani ${ }^{1}$ (D) $\cdot$ Fayaz Shaik Mahammad $^{2} \cdot$ Alex Joseph $^{3}$
}

Received: 22 August 2021 / Revised: 24 October 2021 / Accepted: 25 October 2021 / Published online: 17 November 2021

(c) The Author(s) 2021

\begin{abstract}
Magic spot synthetases are emerging targets to overcome persistence caused by stringent response. The 'stringent response' is a bacterial stress survival mechanism, which results in the accumulation of alarmones (also called Magic spots) leading to the formation of dormant persister cells. These 'sleeper cells' evade antibiotic treatment and could result in relapse of infection. This review broadly investigates the phenomenon of stringent response and persistence, and specifically discusses the distribution, classification, and nomenclature of proteins such as Rel/SpoT homologs (RSH), responsible for alarmone synthesis. The authors further explain the relevance of RSH as potential drug targets to break the dormancy of persister cells commonly seen in biofilms. One of the significant factors that initiate alarmone synthesis is nutrient deficiency. In a starved condition, ribosome-associated RSH detects deacylated tRNA and initiates alarmone synthesis. Accumulation of alarmones has a considerable effect on bacterial physiology, virulence, biofilm formation, and persister cell formation. Preventing alarmone synthesis by inhibiting RSH responsible for alarmone synthesis will prevent or reduce persister cells' formation. Magic spot synthetases are thus potential targets that could be explored to overcome persistence seen in biofilms.
\end{abstract}

Keywords Alarmone synthesis $\cdot$ Bacterial magic spots $\cdot$ Rel/SpoT homologs $\cdot$ Stringent response $\cdot$ Bacterial stress survival mechanism $\cdot$ Wake persister cells

\section{Introduction}

"It is not the strongest of the species that survives; it is the one that is the most adaptable to change"—Charles Darwin

Communicated by Michael Polymenis.

The opinions expressed in this article are the authors' personal views and do not represent that of their affiliated organizations.

Jesil Mathew Aranjani

jesilmathew@gmail.com

1 Department of Pharmaceutical Biotechnology, Manipal College of Pharmaceutical Sciences, Manipal Academy of Higher Education, Manipal, Udupi, Karnataka 576104, India

2 Department of Biotechnology, Manipal Institute of Technology, Manipal Academy of Higher Education, Manipal, Udupi, Karnataka 576104, India

3 Department of Pharmaceutical Chemistry, Manipal College of Pharmaceutical Sciences, Manipal Academy of Higher Education, Manipal, Udupi, Karnataka 576104, India
All organisms must adapt to their environment to survive. Bacteria are endowed with several regulatory systems to adapt to an ever-changing environment around them. Some of these systems work at a transcriptional level, while others operate at a non-transcriptional level. Central longterm survival mechanisms exhibited by bacteria are stringent response (Irving et al. 2020), toxin-antitoxin system (Song and Wood 2020), SOS response (Podlesek and Bertok 2020), and oxidative stress response mechanism (Hong et al. 2019). Of these mechanisms, the stringent response is a pleiotropic stress response mechanism that helps bacteria survive during starvation (amino acids, fatty acids, or carbon sources). The stringent response results in the accumulation of bacterial second messengers called alarmones, and this accumulation results in drastic alteration in the bacterial physiology and metabolism needed for stress survival (Irving et al. 2020). The phenomenon of stringent response was discovered in E. coli by Stent and Brenner (1961) (3). Bacterial second messengers called alarmones or magic spots mediate stringent response. Cashel and Gallant (1969) first identified bacterial magic spots in 1969 . They found two new spots in the thin layer chromatogram of acid-soluble metabolites 
of a stringent $E$. coli culture. The newly identified compounds were christened as Magic spot I and Magic spot II (MS I and MS II). Later these compounds were identified as guanosine $3^{\prime}$-diphosphate $5^{\prime}$-triphosphate (pppGpp) and guanosine $3^{\prime}$-diphosphate $5^{\prime}$-diphosphate (ppGpp) nucleotides. Recently, a third member of this group, guanosine 3'-diphosphate 5'-monophosphate (pGpp), has been confirmed (Yang et al. 2020a). All of them have been together referred to as (pp)pGpp. pppGpp, ppGpp, and pGpp are formed from GTP, GDP, and GMP, respectively, by transferring pyrophosphate moieties from ATP. The transfer of pyrophosphate groups occurs from ATP to the 3'-OH group of ribose of either GTP, GDP, or GMP. pGpp is also formed by hydrolysis of (p)ppGpp by the action of NahA enzyme in Bacillus species (Yang et al. 2020b). Figure 1 shows the formation of pppGpp from ATP and GTP. During the normal growth cycle of bacteria, (p)ppGpp is produced at a basal level and plays important role in maintaining GTP homeostasis, translation, transcription, and energy generation (Gaca et al. 2015a, b; Steinchen et al. 2020). Basal levels of alarmones are also required for expression of virulence factors and antibiotic tolerance (Abranches et al. 2009, 2006; Silva and Benitez 2006). During stress, over production of alarmones occur. This toxic accumulation of high levels (p) ppGpp during stress leads to a physiologically inactive state called persistence.

Bacterial recalcitrance to antibiotics is often attributed to genotypic resistance. However, phenotypic states such as persistence and tolerance can also contribute to antimicrobial tolerance, especially in chronic infections involving biofilms. These antibiotic-tolerant cells are called persisters. Persisters are a small subpopulation (0.001-1\% of the total population) of antibiotic-tolerant but not resistant microbes (Balaban et al. 2019), usually protected deep within biofilms (Dewachter et al. 2019). Hobby et al. (1942) found that penicillin could kill only multiplying cells. Bigger (1944) confirmed persistence in 1944. In his study, he found that a subpopulation of Staphylococcus aureus had become tolerant to the antibiotic penicillin. The slow dividing nature of persister cells makes them tolerant to most antibiotics that target metabolically active cells (Girgis et al. 2012). Persistence can be triggered (type 1) or spontaneous (type 2). Type 1 persistence is initiated by a stress, like starvation or presence of an antibiotic or high cell density or presence of immune factors. Spontaneous persistence is seen in steady-state cultures and is less common than triggered persistence (Balaban et al. 2019). Triggered persistence is not a permanent phenomenon. Persister cells will revert to their usual selves whenever the persistence inducing stress (starvation, antimicrobials, oxygen depletion, thermal shock, $\mathrm{pH}$ variations, etc.) is removed. Persisters are considered as a subpopulation of tolerant bacteria and persistence is also known as heterotolerance (Balaban et al. 2019). Therefore, understanding the mechanism of persister cell formation and ways to prevent the formation or break the dormancy of already formed persister cell will be helpful in devising strategies for the prevention of chronic bacterial infections. The proteins responsible for alarmone synthesis may be potential targets to break the phenomenon of bacterial persistence. These proteins belong to a superclass of enzymes called RelA/SpoT homologs (RSH) or alarmone synthetases or magic spot synthetases.

This review investigates the phenomenon of stringent response and persistence; specifically discusses the distribution, classification, and nomenclature of proteins such as Rel/SpoT homologs (RSH); and explains the mechanisms by which alarmones can be targeted to eliminate persister cells in biofilms.

\section{Distribution of RSH}

RSH are conserved in almost all bacteria and plant chloroplasts (Braeken et al. 2006, 2008; Masuda et al. 2008). Atkinson et al. analyzed 928 complete bacterial genome sequences and found that $92 \%$ of them carried genes encoding a long RSH. Most bacterial species have at least one RSH enzyme, except planctomycetes, verrucomicrobia, and chlamydiae (Atkinson et al. 2011; Hauryliuk and Atkinson 2017). A long Rel/SpoT homolog in plants was first reported in Arabidopsis thaliana. Van der Biezen et al. reported AtRSH1, 2, and 3, which showed significant homology to bacterial RSH in A. thaliana (Van Der Biezen and Jones 1998; Van Der Biezen et al. 2000). The presence of ppGpp in eukaryotes was first reported in Drosophila melanogaster, and scientists found a small alarmone hydrolase: Mesh1 (metazoan SpoT homolog 1) responsible for the hydrolysis of ppGpp. An analog of SpoT, namely human Mesh1 (hMesh1), has been identified in human cells, which is responsible for the activity of NADPH phosphatase that promotes ferroptosis (Ding et al. 2020). Both Mesh1 and hMesh1 are examples of small alarmone hydrolases. The presence of ppGpp in human cells was confirmed by Ito et al. (2020). However, the protein responsible for ppGpp synthesis in metazoans has yet to be confirmed. Bifunctional Rel is the most widely distributed alarmone synthetase in bacteria, while $\beta$ and $\gamma$-proteobacteria encode Rel A and SpoT (Atkinson et al. 2011). It has been postulated that all RSH had a common ancestor, Rel protein, and gene duplication resulted in their diversification (Atkinson and Hauryliuk 2012). Table 1 gives information about RSH present in some common human pathogens. 


\section{Nomenclature and classification of RSH}

Wendrich et al. (2000) came up with a nomenclature system for the three long RSH present in bacteria. They named RelA as ppGpp synthetase I, SpoT as ppGpp synthetase II, and Rel as ppGpp synthetase III. According to their classification, RelA is monofunctional and synthesizes ppGpp during amino acid starvation. SpoT is responsible for the synthesis of ppGpp during carbon source starvation and is also responsible for the degradation of $\mathrm{ppGpp}$ (bifunctional). Bifunctional Rel can synthesize ppGpp during both amino acid and carbon starvation. It is also responsible for the degradation of ppGpp in a manganesedependent manner.
Atkinson et al. classified RSH into 30 subgroups within 3 main groups: long RSH, small alarmone synthetases (SAS), and small alarmone hydrolases (SAH) (Atkinson et al. 2011). Long RSH have multidomain architecture, while SAS and SAH have a single domain organization (Fig. 2). Rel, RelA, and SpoT are examples of long RSH. The N-terminus end of these proteins carries a SYNTH/Synthetase domain and a hydrolase domain (HD) (Pausch et al. 2020). The C-terminus carries the zinc finger domain (ZFD) and aspartate kinase-chorismate mutase-TyrA (ACT)/RRM (ribosome recognition motif) subdomains. The terminals are connected by a middle region formed of the TGS domain and an alpha-helical (AH) region (Kushwaha et al. 2019a, b). The hydrolase

Fig. 1 Synthesis of pppGpp from ATP and GTP. Synthesis occurs by the transfer of diphosphate from ATP to $3^{\prime}-\mathrm{OH}$ oxygen of GTP. Color indicates the transfer

Table 1 Alarmone synthetases present in common human pathogens<smiles>Nc1nc2c(ncn2[C@@H]2O[C@H](COP(=O)(O)P(=O)(O)P(=O)(O)O)[C@@H](O)[C@H]2O)c(=O)[nH]1</smiles>

Guanosine triphosphate

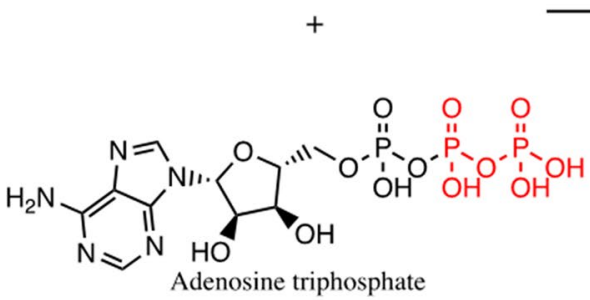<smiles>Nc1nc2c(ncn2[C@@H]2O[C@H](COP(=O)(O)OP(=O)(O)P(=O)(O)O)[C@@H](OP(=O)(O)OP(=O)(O)O)[C@H]2O)c(=O)[nH]1</smiles>

Adenosine monophosphate

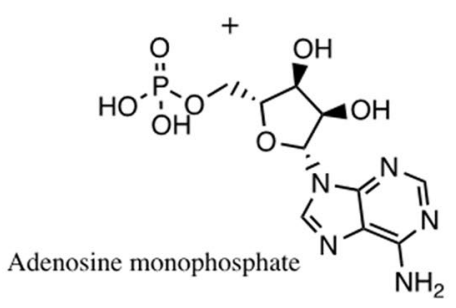

\begin{tabular}{llll}
\hline SI. no & Organism & $\begin{array}{l}\text { Alarmone } \\
\text { synthetase }\end{array}$ & Class \\
\hline 1 & Escherichia coli & RelA & Monofunctional synthetase \\
& & SpoT & Bifunctional RSH \\
2 & Staphylococcus aureus (Siegmund et al. 2021) & Rel & Bifunctional RSH \\
& & yjbm & Small alarmone synthetase \\
& & ywac & Small alarmone synthetase \\
3 & Vibrio cholerae (Dasgupta et al. 2014) & RelV & Small alarmone synthetase \\
& & RelA & Monofunctional synthetase \\
& & SpoT & Bifunctional RSH \\
4 & Bacillus subtilis (Fung et al. 2020) & Rel & Bifunctional RSH \\
& & yjbm & Small alarmone synthetase \\
& & ywac & Small alarmone synthetase \\
5 & Enterococcus faecalis (Gaca et al. 2015a, b) & Rel & Bifunctional RSH \\
& & RelQ & Small alarmone synthetase \\
7 & Mycobacterium tuberculosis (Bag et al. 2014) & Rel & Bifunctional RSH \\
& Pseudomonas aeruginosa (Khakimova et al. 2013) & RelA & Monofunctional synthetase \\
& & SpoT & Bifunctional RSH \\
\hline
\end{tabular}




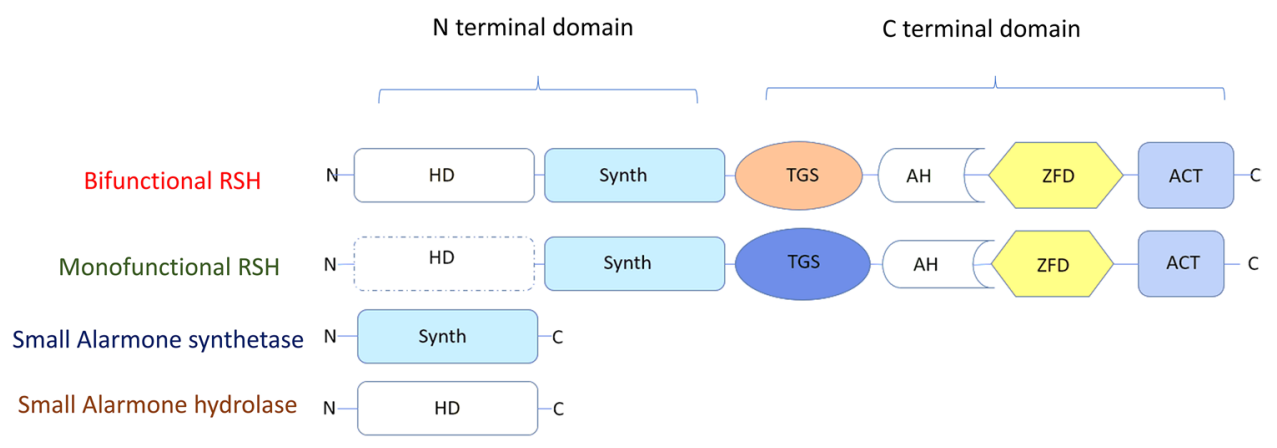

Fig. 2 Domain organization of Rel/SpoT homologs. The hydrolase domain is not functional in monofunctional RSH. Solid shapes represent active domains. The dotted rectangle represents non-functional HD domain. Small alarmone synthetases and small alarmone hydrolases have synthetase and hydrolase domains, respectively. HD hydrolysis domain, Synth synthetase domain, TGS threonyl tRNA synthetase, GTPase, and SpoT, $A H$ alpha helical, $Z F D$ zinc finger domain, ACT aspartate kinase-chorismate mutase-TyrA domain. domain is functional in Rel and SpoT; these enzymes show synthetic and hydrolytic activity (bifunctional) of ppGpp. RelA carries a pseudohydrolase domain and has only alarmone synthetic activity (monofunctional) (Irving et al. 2020). The absence of the HDXXED motif in the HD domain is responsible for the lack of hydrolytic activity of monofunctional RelA (Fig. 3) (Aravind and Koonin 1998). SAS carry only the SYNTH domain and lack all other domains (Jimmy et al. 2019). Examples of SAS are RelQ/YjbM/SAS1/SasB, RelP/YwaC/SAS2/SasA present in firmicutes and RelV present in the genus Vibrio (Hauryliuk and Atkinson 2017; Irving and Corrigan 2018). The three-dimensional structure of SAS resembles the synthetase domain of long RSH (Kushwaha et al. 2019a, b). As the name indicates, SAH carry only the hydrolysis domain. Mesh1 and hMesh1 are examples of SAH. The most studied RSH are the monofunctional RelA of E. coli.

\section{Mechanism of alarmone synthesis (RelA- ribosome-deacylated tRNA complex formation)}

The multidomain protein RelA exists in association with the ribosome. It identifies nutrient deficiency by detecting non-acylated tRNA at the ribosome's A-site (Amino-acyl site) (Kudrin et al. 2018). In the absence of deacyl tRNA, it exists in a closed conformation, and hence there is no synthesis of (p)ppGpp. Usually, the catalytic N-terminal end of the ribosome extends out of the ribosome, while the regulatory $\mathrm{C}$-terminal region is embedded inside the ribosome (Kushwaha et al. 2019a, b). Purified RelA has minimal alarmone synthetic activity in vitro, which suggests the importance of its binding to deacylated tRNA and ribosome (Jenvert and Schiavone 2005). The unbound RelA exists in a closed conformation, devoid of synthetic activity. This closed conformation is caused by the interaction
SP|POAG20|RELA_ECOLI

$\operatorname{tr}|\mathrm{A} 0 \mathrm{~A} 132 \mathrm{P} 4 \mathrm{Q} 3| \mathrm{A} 0 \mathrm{~A} 132 \mathrm{P} 4 \mathrm{Q} 3$ ENTFC

$\operatorname{tr}|A 0 A 069 Q 2 V 6| A 0 A 069 Q 2 V 6 \_P S E A I$

SP|POAG20|RELA ECOLI

$\operatorname{tr}|\mathrm{A} 0 \mathrm{~A} 132 \mathrm{P} 4 \mathrm{Q} 3| \mathrm{A} 0 \mathrm{~A} 132 \mathrm{P} 4 \mathrm{Q} 3$ ENTFC

$\operatorname{tr} \mid$ A0A069Q2V6|A0A069Q2V6_PSEAI

SP|POAG20|RELA_ECOLI

$\operatorname{tr}|\mathrm{A} 0 \mathrm{~A} 132 \mathrm{P} 4 \mathrm{Q} 3| \overline{\mathrm{A}} 0 \mathrm{~A} 132 \mathrm{P} 4 \mathrm{Q} 3$ ENTFC

$\operatorname{tr} \mid$ A0A069Q2V6|A0A069Q2V6_PSEAI $\begin{array}{ll}\text { MVAVRSAHINKAGEFDPEKWIASLG-ITSQKSCECLAETWAYCLQQTQGH--PDASLLLW } & 57 \\ & 52\end{array}$

:. . : . : *. : ${ }^{*}:$. . :

RGVEMVEILSTLSMDIDTLRAALLFPLADANVVSEDVLRESVGKSWVNL IHGVRDMAAIR HPIQVAGILADLKMDPHTVATGFLADVVEDTEITLEDLREEFGDDVAMLVDGVTKLGKIK HPLAVSNILADMHMDHQSLMAAMLKLDVIED GIAKEALTAQFGETVSELVDGVSKLTQMN

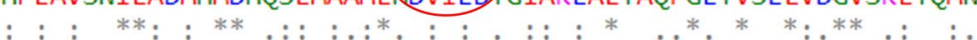

QLKATHTDSVSSEQVDNVRRMLLAMVDDFRCVVIKLAERIAHLREVKDAPEDERVLAAKE -... YKSHEEQLAENHRKMLLAMAQDLRVIMVKLADRLHNMRTLKHLREDKQRRIAQE -...-FETKAEAQAENFQKMAMAMARDIRVILVKLADRLHNMRTLEVLSGEKRRRIAKE : . . .* $::^{*}:^{* *}$, : $^{*}::^{*} *^{*}:^{*}::^{*}:: \quad::$ : $^{*}$
Fig. 3 Result of multiple sequence alignment of HD domains of RelA of Escherichia coli (P0AG20), Rel of Enterococcus faecium (A0A132P4Q3), and, SpoT of Pseudomonas aeruginosa (A0A069Q2V6). It can be found that HDXXED motif (highlighted using a circle) is present in bifunctional Rel and SpoT. The absence of this motif in RelA is the reason behind lack of hydrolytic activity. (Asterisk) Multiple sequence alignment was done using Clustal Omega platform 
(oligomerization) of the RelA CTD with the synthetase domain in NTD (Mechold et al. 2002). The amino acid residues Cys-612, Asp-637, and Cys-638 are essential in this downregulation (Gropp et al. 2001). The formation of disulfide bonds between cysteine residues plays a significant role in this negative regulation of synthetic activity. Yang and Ishiguro (2001) attributed the negative regulatory activity of the C-terminal domain to homodimer formation. They proved that two C-terminal regions: regions from amino acids 455-538 and amino acids 550-682, were responsible for dimerization. In addition to dimer formation, region 550-682 is the main ribosomal binding region of RelA. The CTD of RelA binds to L11 protein and nucleotides 1051-1108 of 23S rRNA of 50S subunit (Agirrezabala et al. 2013). Gropp et al. (2001) proposed that this dimer is enzymatically inactive. Therefore, during the nonstarved condition, RelA exists as an oligomer (specifically a dimer) and is inactive.

Whenever a deacylated tRNA enters the 30S A-site of the ribosome, conformational changes occur, which results in alarmone synthesis (Loveland et al. 2016). The TGS subdomain and $\alpha$-helical region wraps around deacylated tRNA and convert it into a unique conformation called A/R conformation, accommodated at the A-site of the ribosome (Brown et al. 2016; Loveland et al. 2016; Arenz et al. 2016). In this open conformation, the RelA-ribosome-deacylated tRNA complex starts the synthesis of alarmones. According to this popular model, alarmone synthesis requires uncharged tRNA at the A-site of the ribosome bound to mRNA (Haseltine and Block 1973). Wendrich et al. showed that the synthesis of ppGpp was also dependent on the ribosomal protein L11 (Wendrich et al. 2002). The interaction between the L11 protein and the distorted tRNA at the A-site is required to activate the synthetic activity of RelA. They found that ribosomes deficient in the L11 protein, a critical component of the $50 \mathrm{~S}$ ribosome, were inactive in the synthesis of ppGpp. The presence of an optimal concentration of $\mathrm{Mg}^{2+}$ ions is also needed for alarmone synthesis. It was also found that at high concentrations, $\mathrm{Mg}^{2+}$ ions would inhibit alarmone synthesis in bifunctional Rel. Surprisingly this inhibitory effect was not seen in monofunctional RelA. Sajish et al. (2007) proposed that the substitution of the RXKD motif in the synthetase domain of Rel by the EXDD motif in RelA is the reason for this difference. After synthesis of alarmone, RelA gets released from the ribosome, and it hops to the next stalled ribosome to continue alarmone synthesis. Hence, this model is known as the "hopping model" (Wendrich et al. 2002).

English et al. (2011) proposed an "extended hopping model," in which RelA retained its catalytic activity even after dissociation from the ribosome. Winther et al. (2018) came up with an alternative model of complex formation. According to their model, the RelA-deacylated tRNA complex is formed in the cytoplasm, away from the ribosome, and this integrates with the ribosome to create the final complex.

\section{Synthetase domain and ppGpp synthesis}

We have seen the classification of RSH in the preceding section. Despite the differences in domain organization, the active site responsible for alarmone synthesis is commonly seen in all RSH (Patil et al. 2020). ATP, GTP/ GDP, and the catalytic $\mathrm{Mg}^{2+}$ bind to different sites of the synthetase domain to carry out alarmone synthesis. Synthesis and hydrolysis of alarmones are performed by two distinct subdomains present in NTD of Rel protein, namely the synthetase domain and hydrolase domain. The hydrolase domain is inactive in RelA due to the absence of the HDXXED motif (Aravind and Koonin 1998; Irving and Corrigan 2018). The Synthetase/SYNTH subdomain is present in the NTD of RelA of $E$. coli, and it extends from amino acids 181-372 (Brown et al. 2016). Different motifs present in the synthetase domain play a pivotal role in alarmone synthesis. Sajish et al. (2009) studied the importance of conserved motifs EXDD and RXKD present in ppGpp synthetases. Bifunctional RSH has an RXKD motif in the synthetase domain, while monofunctional RSH has an EXDD motif. They found that the specificity of the GTP/GDP substrate (EXDD containing RelA preferred GDP over GTP), response to $\mathrm{Mg}^{2+}$ ions, regulation of catalytic activity by C-terminal domain depended on this motif difference. Patil et al. (2020) proved the catalytic role of 2'-OH of GTP in alarmone synthesis by providing a pivotal hydrogen bond. The binding sites for substrates are present in the synthetase domain. Streptococcus equisimilis $\mathrm{Rel}\left(\mathrm{Rel}_{\mathrm{Seq}}\right)$, the substrate-binding site, comprises amino acid residues Arg241, Lys243, Arg269, Lys297, Tyr299, Lys304, Asn306, Tyr308, His312, Glu323, Arg327, Ala335, and Glu336 (Kushwaha et al. 2019a, b). Figure 4 shows the binding pocket for GDP present in synthetase region of Rel of $S$. equisimilis.

\section{Effects of alarmones on bacterial physiology, virulence, and biofilm formation}

During the stress response, alarmones accumulate inside the bacterial cell. This results in many transcriptional level changes. These changes result in the repression of growth and cell division and activate genes responsible for the biosynthesis of amino acids, acquisition of nutrients, and virulence (Chang et al. 2002; Durfee et al. 2008; Geiger et al. 2010; Chatnaparat et al. 2015; Frank et al. 2014; Vogt et al. 2011). The molecular basis of the stress response varies in Gram-negative and Gram-positive microbes. Different 
A

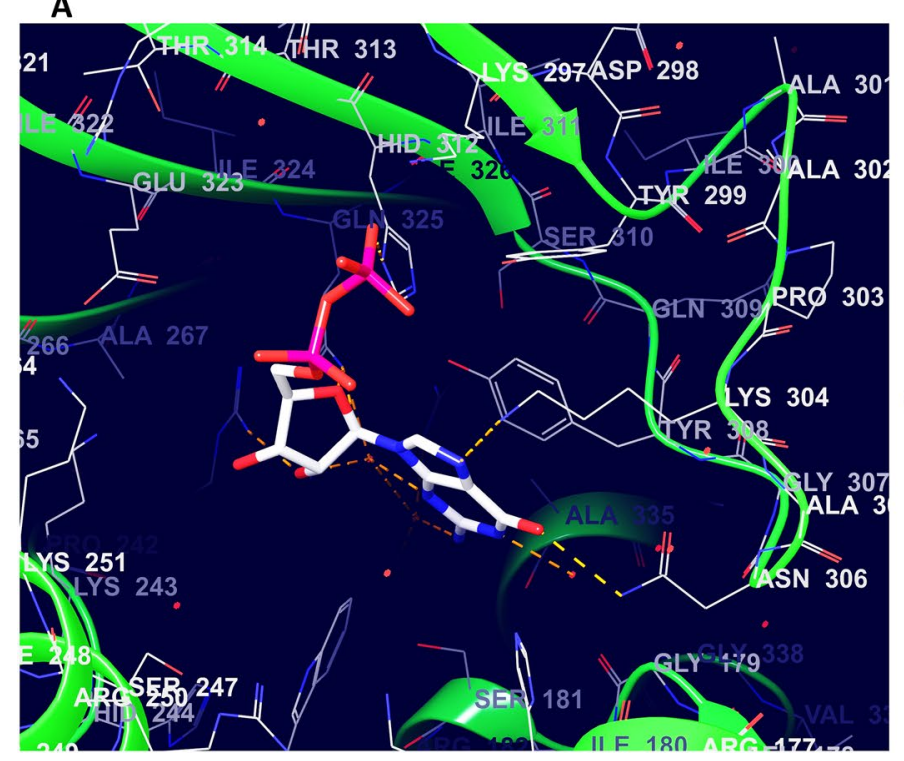

B

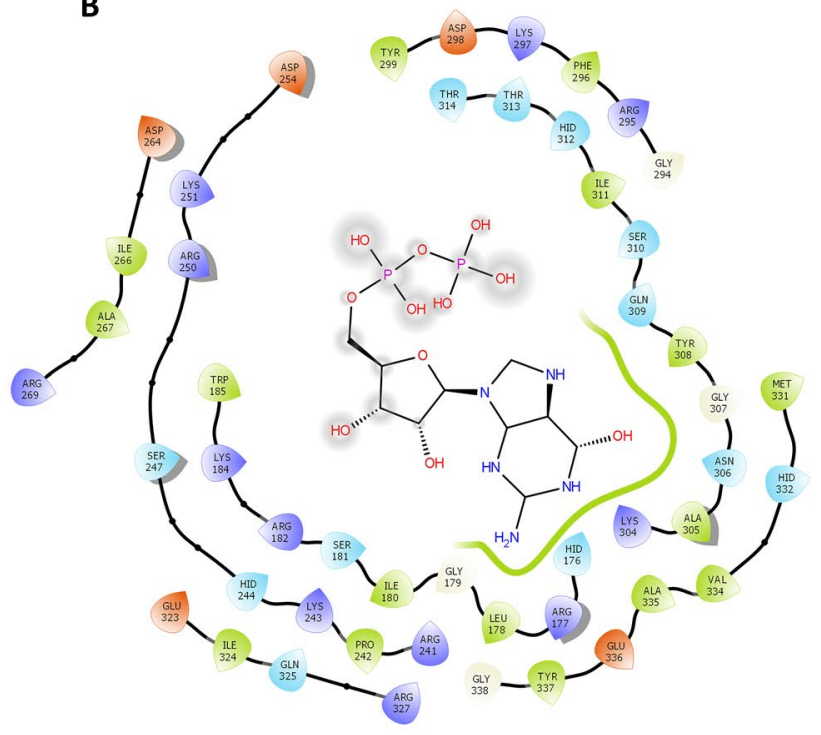

Fig. 4 A The 3D representation of the ligand GDP within the binding pocket of Rel of Streptococcus equisimilis. B The different amino acid residues surrounding the ligand GDP in the synthetase region of Rel of Streptococcus equisimilis. (Asterisk) Structures were taken from Protein data bank and processed using Schrodinger ${ }^{\mathrm{TM}}$ software

vital role in the expression of virulence (Schafhauser et al. 2014). Erickson et al. found that the P. aeruginosa relAmutant strain showed attenuated virulence in a $D$. melanogaster infection model (Erickson et al. 2004). Yersinia pestis relA/spot mutants exhibited decreased amounts of plasmid-encoded virulence proteins associated with the type 3 signaling system (T3SS) (Sun et al. 2009). Aberg et al. (2006) found that ppGpp-deficient uropathogenic $E$. coli (UPEC) failed to produce type 1 fimbriae needed for initial attachment to bladder cells. They also found that such strains had reduced ability to form biofilms. RSH mutant strains of Enterococcus faecalis, E. coli, Streptococcus mutans, and Vibrio cholerae had attenuated virulence and exhibited defective biofilm formation (Balzer and McLean 2011; Lemos et al. 2004; He et al. 2012; Moyed and Bertrand 1983).

\section{Alternative determinants of bacterial persistence}

After the discovery of persistence, many studies were conducted to understand the molecular basis of persistence. A well-studied mechanism for persister formation is the toxin-antitoxin (TA) system (Page and Peti 2016). TA systems play a significant role in the formation of both biofilms and persister cells (Wang and Wood 2011). A toxin is activated following a stress, whose expression inhibits physiological processes. In the HipBA system, stress degrades the antitoxin HipB, which causes expression of toxin HipA. This 
results in suppression of translation factor EF-Tu leading to growth arrest (Schumacher et al. 2009). Kaspy et al. (2013) found that expression of HipA caused phosphorylation of enzyme GltX, which in turn resulted in an accumulation of uncharged tRNA inside cell. This accumulation triggered stringent response. Norton et al. found that the pasTI TA system increased persister formation in extraintestinal pathogenic E. coli (ExPEC), which are significant causative microbes of sepsis, urinary tract infections, and meningitis (Norton and Mulvey 2012). Other TA systems which may have an impact on persister cells' formation are MqsR/Mqs (Kim and Wood 2010), higB, MazF, yafQ and yaeB in $E$. coli (Shah et al. 2006) and MazE, RelE (Lemos et al. 2005). Mutations increase frequency of persister cells' formation. Moyed et al. (1983) found that high persistence mutations (hip mutations such as hipA7) increased the frequency of persister cell formation by 10,000 times in $E$. coli). Wolfson et al identified another high persistence gene HipQ in E. coli. They found that HipQ mutant strain exhibited high persistence in the presence of ampicillin and norfloxacin (Wolfson et al. 1990). Korch et al. (2003) demonstrated that RSH knockouts eliminated persistence in hipA7-mutant strains, which meant that hipA7 mutation induced persistence by over production of alarmones by RSH. Another mechanism that resulted in persistence during the stationary phase was the RpoS-mediated general stress mechanism. RpoS is a gene that encodes a unique sigma factor called RpoS. The accumulation of RpoS inside cells causes the cells to enter the stationary phase (Battesti et al. 2011). Studies have shown that ppGpp is one of the factor controlling the expression of RpoS during stationary phase (Spira and Ospino 2020; Hirsch and Elliott 2002). It is inferred that during starvation there is over production of ppGpp, which causes overexpression of RpoS and subsequent development of persistence. According to Conlon et al. (2016) depletion of ATP could produce persister cells in $S$. aureus. They found that deleting TA modules did not have any effect on persister cell formation in $S$. aureus. This means that toxin-antitoxin systems do not play role in persister formation in Gram-positive $S$. aureus, unlike Gram-negative E. coli. Variations in ATP levels might also play a role in persister cells' generation. As a conclusion, it can be inferred that a multitude of factors contribute to the onset of persistence. More studies are required to conclude which is the most critical mechanism controlling stringent response and how these systems are inter-related.

\section{Magic spot synthetases as a potential drug target to overcome persistence}

To date, two different approaches have been explored to block stringent response mechanisms in bacteria. The first approach involved the discovery of molecules that degrade already formed alarmones. Fuente-Nez et al. synthesized an antibiofilm cationic peptide 1018 (VRLIVAVRIWRR-NH) in 2014, which blocked the stringent response. This peptide caused the degradation of alarmones formed within cells. Peptide 1018 prevented biofilm formation and eradicated biofilms formed in Gram-negative and Gram-positive pathogens such as $P$. aeruginosa, Escherichia coli, Acinetobacter baumannii, Klebsiella pneumoniae, methicillin-resistant $S$. aureus (MRSA), Salmonella typhimurium and Burkholderia cenocepacia (Battesti et al. 2011; Trastoy et al. 2018; de la Fuente-Núñez et al. 2014). In 2015, D-amino acid peptides were made, which were analogs of peptide 1018, which potentiated antibiofilm activities of conventional antibiotics up to almost 64 times (Trastoy et al 2018). These peptides were able to prevent $P$. aeruginosa infections (de la Fuente-Núñez et al. 2014, 2015). A major feature of these peptides is that they acted against biofilms produced by both Gram-positive and Gram-negative microbes, but had a high MIC (minimum inhibitory concentration) against planktonic forms (Fuente-Núñez and Hancock 2015). Peptide 1018 had a MIC of $64 \mu \mathrm{g} / \mathrm{ml}$ against $P$. aeruginosa and $128 \mu \mathrm{g} / \mathrm{ml}$ against $A$. baumannii. Cost of production and stability of peptides in the presence of host proteases are other concerns in developing these peptides as therapeutic agents. Adding to these concerns, a later study proved that peptide 1018 lacked specificity towards ppGpp (Andresen et al. 2016).

The second approach involved designing molecules that inhibited the proteins ( $\mathrm{RSH}$ ) responsible for alarmone synthesis. Most of them were structural modifications of ppGpp. Wexselblatt et al. (2010), synthesized a group of ppGpp analogs known as 'Wexselblatt's Bisphosphonates,' which competitively inhibited RelA activity in vitro. The compound 2-deoxyguanosine-3'-5'-di (methylene bisphosphonate) was found to inhibit RSH in Gram-positive and Gram-negative microbes. Although the introduction of methylene bridges between phosphate groups considerably improved stability, the molecule required $1 \mathrm{mM}$ concentration for $50 \%$ inhibition of $E$. coli RelA in vitro. The hydrophilic nature of molecule rendered it ineffective against live microbes (Beljantseva et al. 2017). Wexselblatt et al. (2012), synthesized a novel antibacterial agent targeting stringent response and named it relacin. It was synthesized by substituting the $3^{\prime}$ and 5' end of ppGpp with glycyl-glycine dipeptides. They studied the inhibitory potential of this compound on GTP pyrophosphokinases from E. coli and B. subtilis. In E. coli, although it showed inhibitory activity in vitro, there was no in vivo activity, which was due to the inability of relacin to cross the bacterial cell wall. But it was found that relacin inhibited both in vitro and in vivo ppGpp synthesis in $B$. subtilis. In B. subtilis, relacin inhibited the formation of biofilm pellicles, as well as the formation of spores (Wexselblatt et al. 2012). When compared to bisphosphonate, relacin had lower hydrophilicity and showed in vivo action against $B$. 
subtilis which was a considerable improvement. Still its potency was not substantial $\left(\mathrm{IC}_{50}=200 \mu \mathrm{M}\right)$ and had no biological activity against Gram-negative microbes (Chau et al. 2021). The quest for more potent molecules started by keeping either relacin or ppGpp as the base molecule.

Wexselblatt et al. (2013), synthesized eight structural analogs of relacin and studied their effects on purified GTP pyrophospho kinases from $E$. coli and $D$. radiodurans. The compound that they named " $2 \mathrm{~d}$ ", which had two glutamylglutamic acid moieties at the $3^{\prime}$ and $5^{\prime}$ end of deoxyribose, showed better in vitro activity than relacin (Wexselblatt et al. 2013)

Syal et al. $(2017 \mathrm{a}, \mathrm{b})$ prepared four synthetic analogs of ppGpp by acetylation and benzoylation reactions. They found that the compound, which was acetylated and benzoylated, inhibited the formation of biofilms and disrupted the formed biofilms in Mycobacterium smegmatis. Syal et al. also found that vitamin $\mathrm{C}$ at high concentration could inhibit in vivo (p)ppGpp formation in M. smegmatis. Vitamin $\mathrm{C}$ in high concentration could stall long-term survival and biofilm formation in M. smegmatis (Syal et al. 2017a, b). Pandit et al found that low concentrations of vitamin $\mathrm{C}$ (upto 30 $\mu \mathrm{M})$ could also hinder biofilm formation by decreasing the synthesis of extra polymeric matrix (Pandit et al. 2017).

In 2019, Dutta et al. virtually screened the GlaxoSmithKline (GSK) compound library that contained 2 million compounds against the Rel protein of Mycobacterium tuberculosis. The compound that showed the most promising inhibitory activity was named "X9". It was found to increase the susceptibility of $M$. tuberculosis to Isonicotinic acid hydrazide (INH) treatment. This showed the potential of Rel inhibitors as potential re-sensitizers of conventional antibiotics (Dutta and Klinkenberg 2019). In Hall et al. (2020) screened the ZINC database using the RelA protein structure from the Protein data bank (PDB ID:5IQR). They came up with two molecules: S3-G1A and S3-G1B, having better docking scores than relacin. Both compounds also showed better in vitro activity. These compounds showed no activity against planktonic cells. From the study, it was also found that the hit compounds reduced the formation of biofilm matrix. An Alamar blue viability assay showed that ampicillin killed more $E$. coli cells when combined with lead molecules.

\section{Conclusion}

Conventional antibiotics kill planktonic cells, but their inability to terminate the dormant persister cells often results in the relapse of infection more severely. To break this phenotypic resistance, new molecules are needed that will eliminate persister cells and inhibit biofilm formation. Magic spot synthetases are such proteins whose inhibition will result both in biofilm inhibition and prevention of persister cell survival. The discovery of such molecules will help in the re-sensitization of antibiotics which are losing their relevance in treating biofilm-related chronic infections. Combining Rel inhibitors with antibiotics will provide a new platform in treating relapsing infections caused by bacterial pathogens.

Funding Open access funding provided by Manipal Academy of Higher Education, Manipal. The authors did not receive any type of funding related to this work.

Data sharing Data sharing is not applicable to this article as no datasets were generated or analyzed.

\section{Declarations}

Conflict of interest The authors declare no conflicts of interest.

Open Access This article is licensed under a Creative Commons Attribution 4.0 International License, which permits use, sharing, adaptation, distribution and reproduction in any medium or format, as long as you give appropriate credit to the original author(s) and the source, provide a link to the Creative Commons licence, and indicate if changes were made. The images or other third party material in this article are included in the article's Creative Commons licence, unless indicated otherwise in a credit line to the material. If material is not included in the article's Creative Commons licence and your intended use is not permitted by statutory regulation or exceeds the permitted use, you will need to obtain permission directly from the copyright holder. To view a copy of this licence, visit http://creativecommons.org/licenses/by/4.0/.

\section{References}

Åberg A, Shingler V, Balsalobre C (2006) (P)PpGpp regulates type 1 fimbriation of Escherichia coli by modulating the expression of the site-specific recombinase FimB. Mol Microbiol 60(6):1520 1533. https://doi.org/10.1111/J.1365-2958.2006.05191.X

Abranches J, Martinez AR, Kajfasz JK, Chavez V, Garsin DA, Lemos JA (2009) The molecular alarmone (p)PpGpp mediates stress responses, vancomycin tolerance, and virulence in Enterococcus faecalis. J Bacteriol 191(7):2248-2256. https://doi.org/10. 1128/JB.01726-08

Agirrezabala X, Fernández IS, Kelley AC, Cartón DG, Ramakrishnan V, Valle M (2013) The ribosome triggers the stringent response by RelA via a highly distorted TRNA. EMBO Rep 14(9):811816. https://doi.org/10.1038/embor.2013.106

Anderson BW, Hao A, Satyshur KA, Keck JL, Wang JD (2020) Molecular mechanism of regulation of the purine salvage enzyme XPRT by the alarmones pppGpp, ppGpp, and pGpp. J Mol Biol 432(14):4108-4126

Anderson BW, Fung DK, Wang JD (2021) Regulatory themes and variations by the stress-signaling nucleotide alarmones $(\mathrm{p})$ ppGpp in bacteria. Annu Rev Genet. https://doi.org/10.1146/ annurev-genet-021821-025827

Andresen L, Tenson T, Hauryliuk V (2016) Cationic bactericidal peptide 1018 does not specifically target the stringent response alarmone (p)PpGpp. Sci Rep. https://doi.org/10.1038/srep36549 
Aravind L, Koonin EV (1998) The HD domain defines a new superfamily of metal-dependent phosphohydrolases. Trends Biochem Sci 23(12):469-472. https://doi.org/10.1016/S0968-0004(98) 01293-6

Arenz S, Abdelshahid M, Sohmen D, Payoe R, Starosta AL, Berninghausen O, Hauryliuk V, Beckmann R, Wilson DN (2016) The stringent factor RelA adopts an open conformation on the ribosome to stimulate PpGpp synthesis. Nucleic Acids Res 44(13):6471-6481. https://doi.org/10.1093/nar/gkw470

Atkinson GC, Hauryliuk V (2012) Evolution and function of the RelA/ SpoT homologue (RSH) proteins. ELS. https://doi.org/10.1002/ 9780470015902.a0023959

Atkinson GC, Tenson T, Hauryliuk V (2011) The RelA/SpoT homolog (RSH) superfamily: distribution and functional evolution of ppGpp synthetases and hydrolases across the tree of life. PLoS ONE. https://doi.org/10.1371/journal.pone.0023479

Bag S, Das B, Dasgupta S, Bhadra RK (2014) Mutational analysis of the (p)ppGpp synthetase activity of the Rel enzyme of Mycobacterium tuberculosis. Arch Microbiol 196(8):575-588

Balaban NQ, Helaine S, Lewis K, Ackermann M, Aldridge B, Andersson DI, Brynildsen MP et al (2019) Definitions and guidelines for research on antibiotic persistence. Nat Rev Microbiol 17(7):441448. https://doi.org/10.1038/s41579-019-0196-3

Balzer GJ, McLean RJC (2011) The stringent response genes RelA and SpoT are important for Escherichia coli biofilms under slow-growth conditions. 48(7):675-80. https://doi.org/10.1139/ W02-060

Battesti A, Majdalani N, Gottesman S (2011) The RpoS-mediated general stress response in Escherichia coli. Annu Rev Microbiol. https://doi.org/10.1146/annurev-micro-090110-102946

Beljantseva J, Kudrin P, Jimmy S, Ehn M, Pohl R, Varik V, Tozawa Y et al (2017) Molecular mutagenesis of PpGpp: turning a RelA activator into an inhibitor. Sci Rep. https://doi.org/10.1038/ SREP41839

Bernardo LM, Johansson LU, Solera D, Skärfstad E, Shingler V (2006) The guanosine tetraphosphate (PpGpp) alarmone, DksA and promoter affinity for RNA polymerase in regulation of sigmadependent transcription. Mol Microbiol 60(3):749-764. https:// doi.org/10.1111/J.1365-2958.2006.05129.X

Biezen EA, Der V, Sun J, Coleman MJ, Bibb MJ, Jones JDG (2000) Arabidopsis RelA/SpoT homologs implicate (p)PpGpp in plant signaling. Proc Natl Acad Sci USA 97(7):3747-3752. https://doi. org/10.1073/pnas.97.7.3747

Bigger JW (1944) Treatment of staphylococcal infections with penicillin by intermittent sterilisation. Lancet 244(6320):497-500. https://doi.org/10.1016/S0140-6736(00)74210-3

Braeken K, Moris M, Daniels R, Vanderleyden J, Michiels J (2006) New horizons for (p)ppGpp in bacterial and plant physiology. Trends Microbiol 14(1):45-54. https://doi.org/10.1016/j.tim. 2005.11.006

Brown A, Fernández IS, Gordiyenko Y, Ramakrishnan V (2016) Ribosome-dependent activation of stringent control. Nature 534(7606):277-280. https://doi.org/10.1038/nature17675

Cashel M, Gallant N (1969) “() 1969 Nature Publishing Group”. Two compounds implicated in the function of the RC gene of Escherichia coli. Nature 224:838-841

Chang D-E, Smalley DJ, Conway T (2002) Gene expression profiling of Escherichia coli growth transitions: an expanded stringent response model. Mol Microbiol 45(2):289-306. https://doi.org/ 10.1046/J.1365-2958.2002.03001.X

Chatnaparat T, Li Z, Korban SS, Zhao Y (2015) The stringent response mediated by (p)ppGpp is required for virulence of Pseudomonas syringae Pv. tomato and its survival on tomato. 28(7):776-89. https://doi.org/10.1094/MPMI-11-14-0378-R

Chau NY, Elizabeth SA, Whitney JC, Coombes BK (2021) Emerging and divergent roles of pyrophosphorylated nucleotides in bacterial physiology and pathogenesis. PLoS Pathog 17(5):e1009532. https://doi.org/10.1371/JOURNAL.PPAT. 1009532

Conlon BP, Rowe SE, Gandt AB, Nuxoll AS, Donegan NP, Zalis EA, Clair G, Adkins JN, Cheung AL, Lewis K (2016) Persister formation in Staphylococcus aureus is associated with ATP depletion. Nat Microbiol 1(5):1-7. https://doi.org/10.1038/nmicrobiol. 2016.51

Dalebroux ZD, Svensson SL, Gaynor EC, Swanson MS (2010) PpGpp conjures bacterial virulence. Microbiol Mol Biol Rev 74(2):171. https://doi.org/10.1128/MMBR.00046-09

Dasgupta S, Basu P, Pal RR, Bag S, Bhadra RK (2014) Genetic and mutational characterization of the small alarmone synthetase gene relV of Vibrio cholerae. Microbiology 160(9):1855-1866

de la Fuente-Núñez C, Hancock REW (2015) Using anti-biofilm peptides to treat antibiotic-resistant bacterial infections. Postdoc J 3(2): 1 ((/pmc/articles/PMC4994992/))

de la Fuente-Núñez C, Reffuveille F, Haney EF, Straus SK, Hancock REW (2014) Broad-spectrum anti-biofilm peptide that targets a cellular stress response. PLoS Pathog. https://doi.org/10.1371/ journal.ppat.1004152

de la Fuente-Núñez C, Reffuveille F, Mansour SC, ReckseidlerZenteno SL, Hernández D, Brackman G, Coenye T, Hancock REW (2015) D-enantiomeric peptides that eradicate wild-type and multidrug-resistant biofilms and protect against lethal Pseudomonas aeruginosa infections. Chem Biol 22(2):196205. https://doi.org/10.1016/j.chembiol.2015.01.002

Dewachter L, Fauvart M, Michiels J (2019) Bacterial heterogeneity and antibiotic survival: understanding and combatting persistence and heteroresistance. Mol Cell 76(2):255-267. https:// doi.org/10.1016/j.molcel.2019.09.028

Ding CK, Cornelia JR, Sun T, Jianli Wu, Chen PH, Lin CC, Yang WH et al (2020) MESH1 is a cytosolic NADPH phosphatase that regulates ferroptosis. Nat Metab 2(3):270-277. https://doi. org/10.1038/s42255-020-0181-1

Durfee T, Hansen AM, Zhi H, Blattner FR, Ding JJ (2008) Transcription profiling of the stringent response in Escherichia coli. J Bacteriol 190(3):1084-1096. https://doi.org/10.1128/ JB.01092-07

Dutta NK, Klinkenberg LG, Vazquez MJ, Segura-Carro D, Colmenarejo G, Ramon F, Rodriguez-Miquel B et al (2019) Inhibiting the stringent response blocks mycobacterium tuberculosis entry into quiescence and reduces persistence. Sci Adv 5(3):2104. https://doi.org/10.1126/sciadv.aav2104

English BP, Hauryliuk V, Sanamrad A, Tankov S, Dekker NH, Elf J (2011) Single-molecule investigations of the stringent response machinery in living bacterial cells. Proc Natl Acad Sci USA 108(31):12573-12574. https://doi.org/10.1073/pnas.1102255108

Erickson DL, Louise Lines J, Pesci EC, Venturi V, Storey DG (2004) Pseudomonas aeruginosa RelA contributes to virulence in Drosophila melanogaster. Infect Immun 72(10):5638. https://doi.org/ 10.1128/IAI.72.10.5638-5645.2004

Frank KL, Colomer-Winter C, Grindle SM, Lemos JA, Schlievert PM, Dunny GM (2014) Transcriptome analysis of Enterococcus faecalis during mammalian infection shows cells undergo adaptation and exist in a stringent response state. PLoS ONE 9(12):e115839. https://doi.org/10.1371/JOURNAL.PONE.0115839

Fung DK, Yang J, Stevenson DM, Amador-Noguez D, Wang JD (2020) Small alarmone synthetase SasA expression leads to concomitant accumulation of pGpp, ppApp, and AppppA in Bacillus subtilis. Front Microbiol 11:2083

Gaca AO, Colomer-Winter C, Lemos JA (2015a) Many means to a common end: the intricacies of (p)PpGpp metabolism and its control of bacterial homeostasis. J Bacteriol 197(7):1146-1156. https://doi.org/10.1128/JB.02577-14 
Gaca AO, Kudrin P, Colomer-Winter C, Beljantseva J, Liu K, Anderson B, Wang JD, Rejman D, Potrykus K, Cashel M, Hauryliuk V (2015b) From (p) ppGpp to (pp) pGpp: characterization of regulatory effects of pGpp synthesized by the small alarmone synthetase of Enterococcus faecalis. J Bacteriol 197(18):2908-2919

Geiger T, Goerke C, Fritz M, Schäfer T, Ohlsen K, Liebeke M, Lalk M, Wolz C (2010) Role of the (p)PpGpp synthase RSH, a RelA/ SpoT homolog, in stringent response and virulence of Staphylococcus aureus. Infect Immun 78(5):1873-1883. https://doi.org/ 10.1128/IAI.01439-09

Girgis HS, Harris K, Tavazoie S (2012) Large mutational target size for rapid emergence of bacterial persistence. Proc Natl Acad Sci USA 109(31):12740-12745. https://doi.org/10.1073/pnas.12051 24109

Gropp M, Strausz Y, Gross M, Glaser G (2001) Regulation of Escherichia coli RelA requires oligomerization of the C-terminal domain. J Bacteriol 183(2):570-579. https://doi.org/10.1128/ JB.183.2.570-579.2001

Hall DC, Król JE, Cahill JP, Ji HF, Ehrlich GD (2020) The development of a pipeline for the identification and validation of smallmolecule Rela inhibitors for use as anti-biofilm drugs. Microorganisms 8(9):1-17. https://doi.org/10.3390/microorganisms8 091310

Haseltine WA, Block R (1973) Synthesis of guanosine tetra and pentaphosphate requires the presence of a codon specific, uncharged transfer ribonucleic acid in the acceptor site of ribosomes. Proc Natl Acad Sci USA 70(5):1564-1568. https://doi.org/10.1073/ pnas.70.5.1564

Hauryliuk V, Atkinson GC (2017) Small alarmone synthetases as novel bacterial RNA-binding proteins. 14(12):1695-99. https://doi.org/ 10.1080/15476286.2017.1367889

He H, Cooper JN, Mishra A, Raskin DM (2012) Stringent response regulation of biofilm formation in Vibrio cholerae. J Bacteriol 194(11):2962-2972. https://doi.org/10.1128/JB.00014-12

Hengge R (2014) The general stress response in gram-negative bacteria. Bact Stress Responses. https://doi.org/10.1128/9781555816 841.ch15

Hirsch M, Elliott T (2002) Role of PpGpp in RpoS stationary-phase regulation in Escherichia coli. J Bacteriol 184(18):5077. https:// doi.org/10.1128/JB.184.18.5077-5087.2002

Hobby GL, Meyer K, Chaffee E (1942) Observations on the mechanism of action of penicillin. Proc Soc Exp Biol Med 50(2):277-280 ((Issue published: June 1, 1942))

Hong Y, Zeng J, Wang X, Drlica K, Zhao X (2019) Post-stress bacterial cell death mediated by reactive oxygen species. Proc Natl Acad Sci 116(20):10064-10071. https://doi.org/10.1073/PNAS. 1901730116

Irving SE, Corrigan RM (2018) Triggering the stringent response: signals responsible for activating (p)PpGpp synthesis in bacteria. Microbiology (united Kingdom) 164(3):268-276. https://doi.org/ 10.1099/mic.0.000621

Irving SE, Choudhury NR, Corrigan RM (2020) The stringent response and physiological roles of (Pp)PGpp in bacteria. Nat Rev Microbiol. https://doi.org/10.1038/s41579-020-00470-y

Ito D, Kawamura H, Oikawa A, Ihara Y, Shibata T, Nakamura N, Asano T, Kawabata S-I, Suzuki T, Masuda S (2020) PpGpp functions as an alarmone in metazoa. Commun Biol 3(1):1-11. https://doi. org/10.1038/s42003-020-01368-4

JenvertSchiavone RMKLH (2005) Characterization of the TRNA and ribosome-dependent PppGpp-synthesis by recombinant stringent factor from Escherichia coli. FEBS J 272(3):685-695. https:// doi.org/10.1111/j.1742-4658.2004.04502.x

Jimmy S, Saha CK, Stavropoulos C, Garcia-Pino A, Hauryliuk V, Atkinson GC (2019) Discovery of small alarmone synthetases and their inhibitors as toxin-antitoxin loci. BioRxiv 575399
Kaspy I, Rotem E, Weiss N, Ronin I, Balaban NQ, Glaser G (2013) HipA-mediated antibiotic persistence via phosphorylation of the glutamyl-TRNA-synthetase. Nat Commun 4(1):1-7. https://doi. org/10.1038/ncomms4001

Khakimova M, Ahlgren HG, Harrison JJ, English AM, Nguyen D (2013) The stringent response controls catalases in Pseudomonas aeruginosa and is required for hydrogen peroxide and antibiotic tolerance. J Bacteriol 195(9):2011-2020

Kim Y, Wood TK (2010) Toxins Hha and CspD and small RNA regulator Hfq are involved in persister cell formation through MqsR in Escherichia coli. Biochem Biophys Res Commun 391(1):209213. https://doi.org/10.1016/J.BBRC.2009.11.033

Korch SB, Henderson TA, Hill TM (2003) Characterization of the HipA7 allele of Escherichia coli and evidence that high persistence is governed by (p)ppGpp synthesis. Mol Microbiol 50(4):1199-1213. https://doi.org/10.1046/J.1365-2958.2003. 03779.X

Kriel A, Bittner AN, Kim SH, Liu K, Tehranchi AK, Zou WY, Rendon S, Chen R, Tu BP, Wang JD (2012) Direct regulation of GTP homeostasis by (p)PpGpp: a critical component of viability and stress resistance. Mol Cell 48(2):231. https://doi.org/10.1016/J. MOLCEL.2012.08.009

Kudrin P, Dzhygyr I, Ishiguro K, Beljantseva J, Maksimova E, Oliveira SRA, Varik V et al (2018) The ribosomal A-Site finger is crucial for binding and activation of the stringent factor RelA. Nucleic Acids Res 46(4):1973-1983. https://doi.org/10.1093/NAR/ GKY023

Kushwaha GS, Bange G, Bhavesh NS (2019a) Interaction studies on bacterial stringent response protein RelA with uncharged TRNA provide evidence for its prerequisite complex for ribosome binding. Curr Genet 65(5):1173-1184. https://doi.org/10. 1007/s00294-019-00966-y

Kushwaha GS, Oyeyemi BF, Bhavesh NS (2019b) Stringent response protein as a potential target to intervene persistent bacterial infection. Biochimie 165:67-75. https://doi.org/10.1016/j.biochi.2019.07.006

Lemos JA, Brown TA, Burne RA (2004) Effects of RelA on key virulence properties of planktonic and biofilm populations of Streptococcus mutans. Infect Immun 72(3):1431-1440. https://doi.org/ 10.1128/IAI.72.3.1431-1440.2004

Lemos JA, Brown TA, Abranches J, Burne RA (2005) Characteristics of Streptococcus mutans strains lacking the MazEF and RelBE toxin-antitoxin modules. FEMS Microbiol Lett 253(2):251-257. https://doi.org/10.1016/J.FEMSLE.2005.09.045

Loveland AB, Bah E, Madireddy R, Zhang Y, Brilot AF, Grigorieff N, Korostelev AA (2016) Ribosome.RelA structures reveal the mechanism of stringent response activation. Elife 5(JULY):1-23. https://doi.org/10.7554/eLife.17029

Masuda S, Tozawa Y, Ohta H (2008) Possible targets of 'magic spots' in plant signalling. 3(11):1021-23. https://doi.org/10.4161/PSB. 6766

Mechold U, Murphy H, Brown L, Cashel M (2002) Intramolecular regulation of the opposing (p)PpGpp catalytic activities of RelSeq, the Rel/Spo enzyme from Streptococcus equisimilis. J Bacteriol 184(11):2878-2888. https://doi.org/10.1128/JB.184. 11.2878-2888.2002

Moyed HS, Bertrand KP (1983) HipA, a newly recognized gene of Escherichia coli $\mathrm{K}-12$ that affects frequency of persistence after inhibition of murein synthesis. J Bacteriol 155(2):768-775. https://doi.org/10.1128/jb.155.2.768-775.1983

Norton JP, Mulvey MA (2012) Toxin-antitoxin systems are important for niche-specific colonization and stress resistance of uropathogenic Escherichia coli. PLoS Pathog 8(10):e1002954. https://doi. org/10.1371/journal.ppat.1002954

Page R, Peti W (2016) Toxin-antitoxin systems in bacterial growth arrest and persistence. Nat Chem Biol 12(4):208-214 
Pandit S, Ravikumar V, Abdel-Haleem AM, Derouiche A, Mokkapati VRSS, Sihlbom C, Mineta K et al (2017) Low concentrations of vitamin $\mathrm{C}$ reduce the synthesis of extracellular polymers and destabilize bacterial biofilms. Front Microbiol. https://doi.org/ 10.3389/FMICB.2017.02599

Patil PR, Vithani N, Singh V, Kumar A, Prakash B (2020) A revised mechanism for (p)PpGpp synthesis by Rel proteins: the critical role of the 29-OH of GTP. J Biol Chem 295(37):12851-12867. https://doi.org/10.1074/jbc.RA120.013636

Pausch P, Abdelshahid M, Steinchen W, Schäfer H, Gratani FL, Freibert SA, Wolz C, Turgay K, Wilson DN, Bange G (2020) Structural basis for regulation of the opposing (p)PpGpp synthetase and hydrolase within the stringent response orchestrator Rel. Cell Rep 32(11):108157. https://doi.org/10.1016/J.CELREP. 2020.108157

Podlesek Z, Bertok DŽ (2020) The DNA damage inducible SOS response is a key player in the generation of bacterial persister cells and population wide tolerance. Front Microbiol. https://doi. org/10.3389/FMICB.2020.01785

Ross W, Sanchez-Vazquez P, Chen AY, Lee JH, Burgos HL, Gourse RL (2016) PpGpp binding to a site at the RNAP-DksA interface accounts for its dramatic effects on transcription initiation during the stringent response. Mol Cell 62(6):811-823. https://doi.org/ 10.1016/J.MOLCEL.2016.04.029

Sajish M, Tiwari D, Rananaware D, Nandicoori VK, Prakash B (2007) A charge reversal differentiates (p)PpGpp synthesis by monofunctional and bifunctional Rel proteins. J Biol Chem 282(48):34977-34983. https://doi.org/10.1074/jbc.M704828200

Sajish M, Kalayil S, Verma SK, Nandicoori VK, Prakash B (2009) The significance of EXDD and RXKD motif conservation in Rel proteins. J Biol Chem 284(14):9115-9123. https://doi.org/ 10.1074/jbc.M807187200

Schafhauser J, Lepine F, McKay G, Ahlgren HG, Khakimova M, Nguyen D (2014) The stringent response modulates 4-hydroxy2-alkylquinoline biosynthesis and quorum-sensing hierarchy in Pseudomonas aeruginosa. J Bacteriol 196(9):1641-1650. https:// doi.org/10.1128/JB.01086-13

Schumacher MA, Piro KM, Xu W, Hansen S, Lewis K, Brennan RG (2009) Molecular mechanisms of HipA-mediated multidrug tolerance and its neutralization by HipB. Science (new York, N.y.) 323(5912):396-401. https://doi.org/10.1126/SCIENCE.1163806

Shah D, Zhang Z, Khodursky AB, Kaldalu N, Kurg K, Lewis K (2006) Persisters: a distinct physiological state of $E$. coli. BMC Microbiol 6(1):1-9. https://doi.org/10.1186/1471-2180-6-53

Siegmund A, Afzal MA, Tetzlaff F, Keinhörster D, Gratani F, Paprotka $\mathrm{K}$, Westermann $\mathrm{M}$ et al (2021) Intracellular persistence of Staphylococcus aureus in endothelial cells is promoted by the absence of phenol-soluble modulins. Virulence 12(1):1186-1198

Silva AJ, Benitez JA (2006) A Vibrio cholerae relaxed (RelA) mutant expresses major virulence factors, exhibits biofilm formation and motility, and colonizes the suckling mouse intestine. J Bacteriol 188(2):794-800. https://doi.org/10.1128/JB.188.2.794-800.2006

Song S, Wood TK (2020) A primary physiological role of toxin/antitoxin systems is phage inhibition. Front Microbiol. https://doi. org/10.3389/FMICB.2020.01895

Spira B, Ospino K (2020) Diversity in E. coli (p)PpGpp levels and its consequences. Front Microbiol. https://doi.org/10.3389/FMICB. 2020.01759

Steinchen W, Zegarra V, Bange G (2020) (P)PpGpp: magic modulators of bacterial physiology and metabolism. Front Microbiol. https:// doi.org/10.3389/FMICB.2020.02072

Stent GS, Brenner S (1961) A genetic locus for the regulation of ribonucleic acid synthesis. Proc Natl Acad Sci USA 47(12):2005-2014

Sun W, Roland KL, Branger CG, Kuang X, Curtiss Roy III (2009) The role of RelA and SpoT in Yersinia pestis KIM5+ pathogenicity.
PLoS ONE 4(8):e6720. https://doi.org/10.1371/JOURNAL. PONE.0006720

Syal K, Bhardwaj N, Chatterji D (2017a) Vitamin C targets (p)PpGpp synthesis leading to stalling of long-term survival and biofilm formation in Mycobacterium smegmatis. FEMS Microbiol Lett. https://doi.org/10.1093/femsle/fnw282

Syal K, Flentie K, Bhardwaj N, Maiti K, Jayaraman N, Stallings CL, Chatterji D (2017b) Synthetic (p)PpGpp analogue is an inhibitor of stringent response in mycobacteria. Antimicrob Agents Chemother. https://doi.org/10.1128/AAC.00443-17

Szalewska-Palasz A, Johansson LUM, Bernardo LMD, Skärfstad E, Stec E, Brännström K, Shingler V (2007) Properties of RNA polymerase bypass mutants: implications for the role of PpGpp and its co-factor DksA in controlling transcription dependent on sigma54. J Biol Chem 282(25):18046-18056. https://doi.org/10. 1074/JBC.M610181200

Trastoy R, Manso T, Fernández-García L, Blasco L, Ambroa A, Pérezdel Molino ML, Bou G, García-Contreras R, Wood TK, Tomás M (2018) Mechanisms of bacterial tolerance and persistence in the gastrointestinal and respiratory environments. Clin Microbiol Rev. https://doi.org/10.1128/CMR.00023-18

Van Der Biezen EA, Jones JDG (1998) Curiosity and a dash of good luck. Curr Biol 8(7):R225-R227. https://doi.org/10.1016/s09609822(98)70144-7

Vogt SL, Green C, Stevens KM, Day B, Erickson DL, Woods DE, Storey DG (2011) The stringent response is essential for Pseudomonas aeruginosa virulence in the rat lung agar bead and Drosophila melanogaster feeding models of infection. Infect Immun 79(10):4094-4104. https://doi.org/10.1128/IAI.00193-11

Wang X, Wood TK (2011) Toxin-antitoxin systems influence biofilm and persister cell formation and the general stress response. Appl Environ Microbiol 77(16):5577-5583

Wendrich TM, Beckering CL, Marahiel MA (2000) Characterization of the RelA/SpoT gene from Bacillus stearothermophilus. FEMS Microbiol Lett 190(2):195-201. https://doi.org/10.1016/S03781097(00)00335-9

Wendrich TM, Blaha G, Wilson DN, Marahiel MA, Nierhaus KH (2002) Dissection of the mechanism for the stringent factor RelA. Mol Cell 10(4):779-788. https://doi.org/10.1016/S10972765(02)00656-1

Wexselblatt E, Katzhendler J, Saleem-Batcha R, Hansen G, Hilgenfeld R, Glaser G, Vidavski RR (2010) PpGpp analogues inhibit synthetase activity of Rel proteins from gram-negative and grampositive bacteria. Bioorg Med Chem 18(12):4485-4497. https:// doi.org/10.1016/j.bmc.2010.04.064

Wexselblatt E, Oppenheimer-Shaanan Y, Kaspy I, London N, SchuelerFurman O, Yavin E, Glaser G, Katzhendler J, Ben-Yehuda S (2012) Relacin, a novel antibacterial agent targeting the stringent response. PLoS Pathog 8(9):1002925. https://doi.org/10.1371/ journal.ppat.1002925

Wexselblatt E, Kaspy I, Glaser G, Katzhendler J, Yavin E (2013) Design, synthesis and structure-activity relationship of novel relacin analogs as inhibitors of Rel proteins. Eur J Med Chem 70:497-504. https://doi.org/10.1016/j.ejmech.2013.10.036

Winther KS, Roghanian M, Gerdes K (2018) Activation of the stringent response by loading of RelA-TRNA complexes at the ribosomal A-site. Mol Cell 70(1):95-105.e4. https://doi.org/10.1016/j.molcel.2018.02.033

Wolfson JS, Hooper DC, McHugh GL, Bozza MA, Swartz MN (1990) Mutants of Escherichia coli K-12 exhibiting reduced killing by both quinolone and beta-lactam antimicrobial agents. Antimicrob Agents Chemother 34(10):1938. https://doi.org/10.1128/AAC. 34.10 .1938

Yang X, Ishiguro EE (2001) Dimerization of the RelA protein of Escherichia coli. Biochem Cell Biol 79(6):729-736. https://doi. org/10.1139/bcb-79-6-729 
Yang J, Anderson BW, Turdiev A, Turdiev H, Stevenson DM, Amador-Noguez D, Lee VT, Wang JD (2020a) The nucleotide PGpp acts as a third alarmone in bacillus, with functions distinct from those of (p) PpGpp. Nat Commun. https://doi.org/10.1038/ s41467-020-19166-1

Yang J, Anderson BW, Turdiev A, Turdiev H, Stevenson DM, AmadorNoguez D, Lee VT, Wang JD (2020b) Systemic characterization of pppGpp, ppGpp and PGpp targets in bacillus reveals NahA converts (p)PpGpp to PGpp to regulate alarmone composition and signaling. Biorxiv. https://doi.org/10.1101/2020.03.23. 003749 ((2020.03.23.003749))

Publisher's Note Springer Nature remains neutral with regard to jurisdictional claims in published maps and institutional affiliations. 